\title{
Gehirn (und Geist?) nach Tod im Schlachthof
}

Einer Arbeitsgruppe von der Yale School of Medicine ist es gelungen, Gehirne von Schweinen 4 Stunden nach deren Tod durch Schlachtung für bis zu 6 Stunden wiederzubeleben. Die Arbeit wurde am 18 April 2019 im Fachblatt Nature publiziert ( $\triangleright$ Abb. 1) [1] und war von gleich 3 (!) jeweils ausführlichen Kommentaren mit Überlegungen dazu begleitet, was das bedeutet und vor allem, was das bedeuten könnte, wenn man etwas weiterdenkt. Bislang waren nur Philosophen gelegentlich daran interessiert, darüber nachzudenken, was es bedeuten könnte, wenn ein Gehirn in einem Bottich am Leben gehalten wird und selbst darüber nachdenkt. Der US-amerikanische Logiker Hilary Putnam betitelte das erste Kapitel eines seiner bekanntesten Bücher mit dem Titel "Gehirne im Bottich“ (Brains in a vat) und ging darin den Schwierigkeiten und Widersprüchen nach, die sich bei diesen Überlegungen auftun [2].

Neurowissenschaftler gingen solchen Gedanken damals eher nicht nach - zumindest nicht in ihren Publikationen -, weil sie schlicht zu realitätsfern erschienen. Das Gehirn stellt seine Arbeit wenige Sekunden nach der Unterbrechung seiner Sauerstoffzufuhr ein. Wegen seines extrem hohen Energiebedarfs sind die Reserven an Glukose und ATP nach wenigen Minuten erschöpft und die Mitochondrien arbeiten nicht mehr. Aufgrund der dann nicht mehr funktionierenden lonenpumpen kommt es zu einem Abfall des Ruhepotenzials der Zellmembranen, zellschädigender Depolarisationen (Excitotoxizität) und einer Anhäufung von toxischem Glutamat. Dies resultiert in irreversiblen Schäden der Axone und neuronalem Zelltod durch Apoptose und Nekrose.

Zwar lässt sich bis heute nicht genau sagen, wann - auf die Minute genau - dann „der Tod“ eintritt, aber dieses Konzept eines genauen Todeszeitpunkts ist ohnehin naturwissenschaftlich etwa ebenso überholt wie die „freie Willensentscheidung“ (einschließlich deren Zeitpunkt) eines Täters: Das Ganze ist vielmehr ein Prozess, und die Leber stirbt „später“ als das Gehirn und die Haut noch später (weswegen einem Toten noch am nächsten Tag der Bart wachsen kann). Den genauen Todeszeitpunkt eines Menschen gibt es in Naturwissenschaft und Medizin nicht (sondern nur in der Rechtsprechung).

Den in Nature erschienenen Kommentaren zufolge, sollten wir damit beginnen, über dieses Problem neu nachdenken. Nicht weil mit der einen neuen Studie alles frühere Wissen zum Gehirn und dessen Ausfall über Bord geworfen werden muss, sondern weil längst vorhandene Brüche in unserem Weltbild ein Stückchen offensichtlicher geworden sind. Es wurde ein neuer Horizont eröffnet, vor dessen Hintergrund das Knirschen im Gebälk unserer für tragfähig erachteten Wissensstrukturen lauter geworden ist und vor allem künftig noch lauter werden wird. Worum geht es?

Die Neurowissenschaftler in Yale machten sich die Tatsache zunutze, dass ihr Institut nicht weit von einem Schlachthof gelegen ist, in dem Schweinefleisch „produziert“ wird. Die Köpfe der bei der Schlachtung 6 bis 8 Monate alten Tiere sind dabei Abfallprodukt, das keinerlei ethischen Regularien unterliegt - toter Müll eben. Mehr als 300 solcher toter Tierköpfe dienten dazu, zunächst eine Methode zu entwickeln, die Gehirne weitgehend freizulegen und diese dann mit einer eigens entwickelten, an die beiden internen Karotiden sowie den venösen Abfluss angeschlossenen „Herz-Lungen-Maschine“ und einer speziellen Technik bei $37^{\circ} \mathrm{C}$ zu perfundieren und experimentell zu untersuchen. Man verwendete kein Blut, sondern eine Flüssigkeit, die u. a. Hämoglobin und nicht-koagulierende, zytoprotektive Stoffe enthielt, jedoch (abgesehen von einem Ultraschallkontrastmittel) keine zellulären bzw. korpuskulären Bestandteile. Das gesamte System nennen die Autoren „BrainEx“ (BEx).

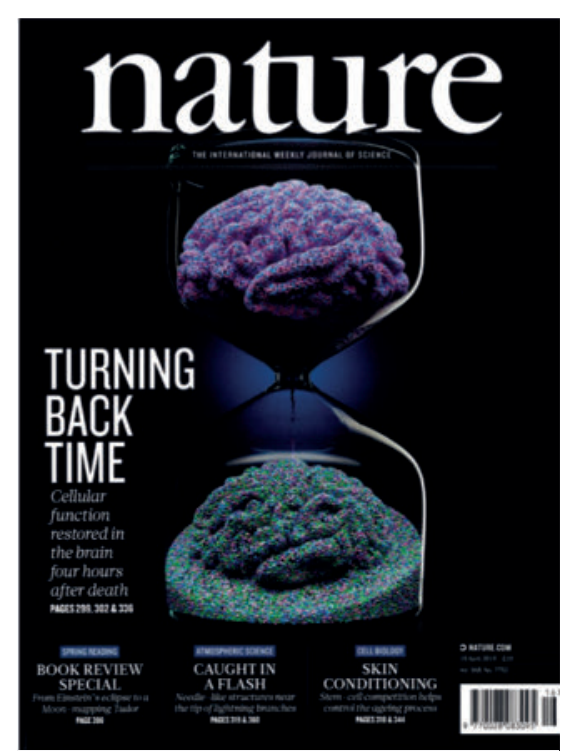

- Abb. 1 Titelseite der Nature-Ausgabe, die den hier diskutierten Artikel enthielt. Die unten in der Sanduhr erneut entstehende Form des Gehirns deutet dessen Überdauern über die Zeit hinweg grafisch-metaphorisch an.

Nachdem die Methode etabliert worden war, wurde die eigentliche Studie an 32 Gehirnen durchgeführt, die in 4 Gruppen - entsprechend 4 verschiedener durchgeführter Prozeduren - eingeteilt wurden: 1. Perfusion mit einer Kontrollflüssigkeit,

2. Perfusion mit der eigens entwickelten BEx-Lösung,

3. Kontrolle ohne Perfusion und Aufarbeitung nach 10 Stunden und

4. Extraktion und kurze Spülung des Gehirns und Aufarbeitung nach einer Stunde.

In den ersten beiden Gruppen begann die Perfusion 4 Stunden nach der Schlachtung (dem „Tod“) des Tieres und wurde für weitere 6 Stunden durchgeführt, wonach die Gehirne makro- und mikroskopisch aufgearbeitet wurden. Es gab also im Grunde eine Behandlungsgruppe (Gruppe 2) und 3 verschiedene Kontrollgruppen.

Während der 6-stündigen Perfusion wurden Experimente mit unterschiedlichen 
Methoden zu verschiedenen Funktionen des Gehirns gemacht.

\section{Dopplersonografie - Perfusion}

Zunächst wurde gezeigt, dass es bei Verwendung von BEx-Lösung tatsächlich zu einer Reperfusion des Gehirns kam, wohingegen die Perfusion mit Kontrolllösung zu einem massiven Gehirnödem, einer Gehirnschwellung und einer Zerstörung von Gehirngewebe führte. Die Perfusion des Gehirns war über den Beobachtungszeitraum zum Stillstand gekommen. Demgegenüber wurde mittels Dopplersonografie nicht nur gezeigt, dass die Perfusion mit BEx-Lösung über den gesamten Zeitraum von 6 Stunden funktionierte. Verabreichte man einen Vasodilatator (Bolusinjektion des Kalziumantagonisten Nimodipin), kam es zu einer dopplersonografisch nachweisbaren Gefäßerweiterung um etwa 40\%. Mit dem BEx-System gelang also nicht nur eine dauerhafte Perfusion, sondern es konnte auch gezeigt werden, dass das Gefäßsystem des Gehirns seine pharmakologische Reagibilität wiedergewonnen hatte.

\section{MRT (T1-gewichtet) - Struktur}

Unter der BEx-Bedingung blieben die anatomischen Strukturen (im Vergleich zu lebenden Gehirnen) intakt, was sich an erhaltener Ventrikelgröße, erhaltenem Kontrast zwischen grauer und weißer Substanz und erhaltenen abgrenzbaren anatomischen Landmarken zeigte. In der Kontrollgruppe ohne Gehirnperfusion (Gruppe 3) sah man hingegen nach 10 Stunden kollabierte Seitenventrikel, einen verminderten Wassergehalt und Gasbildung als Zeichen eines Gehirnzerfalls. Bei Perfusion mit Kontrolllösung (Gruppe 1) kam es hingegen zu vergrößerten Seitenventrikeln, einem erhöhten Wassergehalt des Gehirns und einer Verminderung der MR-Signalintensität.

\section{Histologie - Zytoarchitektur}

Gehirnschnitte von Neokortex, Hippocampus und Kleinhirn zeigten mittels Nissl-Färbung deutliche Unterschiede zwischen den Gruppen. In der BEx-Bedingung (Gruppe 2) zeigte die Histologie intakte Strukturen, wohingegen die nicht perfundierten bzw. nur mit Kontrolllösung perfundierten Gehirne einen deutlichen strukturellen Integritätsverlust zeigten. In ultrastrukturellen Untersuchungen der CA1-Region des Hippocampus, die bekanntermaßen gegenüber Anoxie besonders vulnerabel ist, zeigten die Neuronen in der BEx-Bedingung weniger Anschwellung im Vergleich zur Gruppe 4 mit rascher Aufarbeitung der Gehirne nach Spülung innerhalb von einer Stunde. Auch im präfrontalen und motorischen Neokortex zeigte sich eine erhaltene mikrostrukturelle Integrität, was im Hinblick auf die Betzzellen des Motorkortex, deren Axone ja durchtrennt werden mussten, besonders hervorzuheben ist.

\section{Biochemie - Apoptose, myelinisierte Fasern}

Da die BEx-Perfusionslösung auch einen Hemmstoff der Apoptose (Caspase-Hemmer) enthielt, wundert nicht, dass eine histologische Anfärbung von aktivierter Caspase 3 in Gehirnschnitten des Hippocampus (CA1-region und Gyrus dendatus) eine Verminderung von deren Aktivität in der Gruppe 2 verglichen mit allen anderen Gruppen zeigte. Dies traf auch für die Schnitte aus dem Neokortex zu. Analysen des basischen Myelinproteins zeigten eine Verminderung der Ordnung bzw. Gerichtetheit von Fasern der weißen Substanz in den Gruppen 1 und 3 verglichen mit den Gruppen 2 und 4.

\section{Glia - Inflammation}

Ein ähnliches Ergebnis hatte eine Färbung mit Markern für Astrozyten und Mikroglia: In den Gehirnen ohne Perfusion (Gruppe 3) und mit Perfusion durch Kontrollflüssigkeit (Gruppe 1) fanden sich Anzeichen für Zelluntergang, nicht jedoch in den BEx-perfundierten Gehirnen und den rasch aufgearbeiteten Gehirnen. Um die Funktionstüchtigkeit der genannten Zellen zu untersuchen, wurde lokal der Glia-Aktivator Lipopolysacharid (LPS) im Frontalhirn injiziert, was zu einer Immunantwort in der betreffenden Hirnregion nach 6 Stunden Perfusion im Sinne einer Erhöhung von Interleukin (IL)-1 alpha, IL-1beta, IL-6und IL-8 in Gruppe 2 im Vergleich zu den Gruppen 1 und 4 führte (in Gruppe 3 waren wegen rückläu- figer bzw. fehlender Perfusion während der 6 Stunden entsprechende Messungen nicht möglich). „Insgesamt zeigen diese Befunde, dass die BEx-Perfusion die Astrozyten und Mikrogliazellen nicht nur zahlenmäßig, sondern auch im Hinblick auf ihre inflammatorischen Funktionen aufrechterhalten kann “, fassen die Autoren ihre Ergebnisse zusammen [1, S. 340].

\section{Elektronemikroskopie - Ultrastruktur der Synapsen}

Die elektronenmikroskopische Untersuchung von Hippocampuspräparaten zeigte intakte Synapsen (präsynaptische Vesikel) quantitativ in Gruppen 2 und 4 nicht unterschiedlich, in Gruppen 1 und 3 dagegen auf etwa $50 \%$ reduziert).

\section{Metabolismus}

Messungen der Glukose- und Sauerstoffkonzentrationen in der arteriellen und venösen Perfusionsflüssigkeit (nur Gruppe 2) zeigten einen nach Erwärmung der Gehirne stabilen Sauerstoff- und Glukoseverbrauch an. Auch die venösen Natrium- und Kaliumkonzentrationen stabilisierten sich während der 6-stündigen Perfusion, was ebenfalls eine aktive Natrium-Kalium-Hömöostase und damit einen intakten Metabolismus anzeigt.

\section{Elektrophysiologie in Gehirnschnitten}

Wegen erheblicher Gewebeschäden in den Gehirnen der Gruppen 1 und 3 ließen sich elektrophysiologische Untersuchungen an Neuronen in Gehirnschnitten aus dem Hippocampus (via whole-cell patch-clamp) nur von den BEx-perfundierten Gehirnen durchführen. Hier zeigten sich normale Werte für Membrankapazität, Membranwiderstand, Zeitkonstante und Ruhepotenzial. „Alle abgeleiteten Neuronen reagierten auf überschwellige Depolarisation mit repetitiven Aktionspotentialen und wiesen sowohl schnelle voltage-gated $\mathrm{Na}$ trium-Ionen-Ströme als auch langsame rectifyer Kalium-Ionen-Ströme auf" [1, S. 341]. Auch die Schwelle des Auftretens von Aktionspotenzialen und deren Amplitude waren normal. „Wir fanden also funktio- 
nierende Pyramidenzellen im Hippocampus nach 4 Stunden post mortem begonnener 6-stündiger Gehirnperfusion“, fassen die Autoren zusammen [1, S. 341].

\section{EEG}

Zwanzig intrakranielle direkt auf dem Kortex platzierte Elektroden dienten der Elektrokortikografie (ECoG, eine Art intrakranielles EEG). Es zeigte sich in allen Ableitungen von den BEx-perfundierten Gehirnen eine Null-Linie. „Dies zeigt an, dass Organisation und/oder Summation der synaptischen Aktivität einzelner Neuronen nicht ausreichte, um eine mittels ECOG messbare Aktivität des Netzwerkaktivität zu erzeugen “, kommentieren die Autoren dieses Ergebnis [1, S. 341].

Nach all den wiederhergestellten Funktionen ist dieses letzte Ergebnis - das Gehirn als Ganzes funktioniert nicht mehr (vom Geist gar nicht zu reden!) - zunächst enttäuschend. Möglicherweise handelt es sich dabei jedoch um ein experimentelles Artefakt. Um dies zu verstehen, muss man das Supplement und die Kommentare genau lesen, die sich vor allem auf die durch das Experiment aufgeworfenen ethischen Fragen beziehen.

Darf man das alles überhaupt? - Es gehört zweifellos zu den Verdiensten der Autoren, dass sie so ziemlich jeden und alle gefragt haben, die für eine Antwort zuständig sein könnten: Von der lokalen Ethikkommission bis hin zur Neuroethics Working Group der BRAIN-Initiative der National Institutes of Health (NIH)1. Wie eingangs gesagt, gibt es für den bei der Schlachtung von Tieren entstehenden Abfall keinerlei ethische Regularien. Das Schlachten selbst - möglichst schnell und schmerzfrei etc. - ist dagegen geregelt, und für die Behandlung von Versuchstieren in wissenschaftlichen Experimenten gibt es jede Menge Regeln. Weil

1 In einem Kommentar findet sich hierzu: „The Yale group was conscientious and consulted the local institutional IACUC, Yale bioethicists, NIH programme officers and even the NIH Neuroethics Working Group. The researchers did what they could, and probably more than many would have done, to ensure that they were acting appropriately in a void of ethical analysis on the issue " [3, S. 302; Hervorhebung durch den Autor]. das gesamte Experiment also einerseits erst 4 Stunden nach dem Tod der Tiere begann, aber andererseits darauf abzielte, die Gehirnfunktion wiederherzustellen, wurde es als eine Gefahr betrachtet, dass dies tatsächlich geschehen könnte und den Tieren - durch Wiedererlangung von geistigen Leistungen wie Wahrnehmung oder Schmerzempfindung - Leid zugefügt würde. Um dies zu verhindern wurden der Perfusionslösung nicht nur Blocker von Zelltodprozessen beigemischt, sondern auch Blocker neuronaler Aktivität. Nach der Entnahme der Gehirnproben für die elektrophysiologischen Experimente gab es daher immer auch eine Washout-Phase. „Wenn diese Blocker (während des Experiments) irgendwann entfernt worden wären, hätte das Forschungsteam möglicherweise EEG-Aktivität gefunden“, kann man in einem der Kommentare daher lesen [3, S. 300]. Im Supplement wird darüber hinaus beschrieben, dass man während des gesamten Experiments Anästhetika „zur Hand" hatte und zudem das Gehirn sofort gekühlt hätte, wenn man spontane Hirnstromaktivität festgestellt hätte: „It is remotely conceivable that as the technology develops, the brain may regain the ability to emit global (i. e., ECoG/EEG) signals. However, in the event that this were to occur, we were ready to swiftly implement countermeasures, including, but not limited to, reducing the temperature of the brain in order to diminish metabolic activity, and/or administering general anesthetic agents (which were already at-hand during the perfusion experiments) to maintain an isoelectric ECoG reading. (...) Given the possibilities for inadvertent suffering posed by the restoration and maintenance of remnant awareness, any experimentation aimed at purposefully maintaining a brain with global electrical activity, without the aforementioned interventions to diminish such activity, should be subject to more extensive ethical review“ [4].

Weil man also aus ethischen Gründen sehr vorsichtig war, konnten man gar nicht finden, was eigentlich gefunden werden sollte: die Wiederherstellung der Gehirnfunktion. Und die Autoren schreiben explizit, dass man jetzt weiter experimentieren kann, so weit, wie es die - noch zu erfolgende - Diskussion der ethischen Aspekte eben erlaubt. Die Kommentatoren sprechen durch- aus bereits davon, dass dies auch ein Modell für das funktionierende menschliche Gehirn darstellen könnte und es daher höchste Zeit wird, dass man darüber diskutiert.

In den Kommentaren geht es u. a. um die Frage, was diese Arbeit für die Transplantationsmedizin heute schon bedeutet. Wenn die Frage des Hirntods wieder unklarer erscheint, zumal die Arbeitsgruppe Gehirne für bis zu 36 Stunden „am Leben“ gehalten hat [5], wird dessen Feststellung möglicherweise wieder schwieriger und damit das Transplantieren unwahrscheinlicher. Kostet die Arbeit also womöglich manchem, der auf ein Organ wartet, das Leben. Hierzu eine Zahl aus einem der Kommentare: Im Jahr 2017 verstarben in den USA täglich etwa 18 Patienten auf den Wartelisten für Organe, die ihren Tod hätten verhindern können. Bei uns fehlen ebenfalls Spender. In den Worten der Kommentatoren: „If technologies similar to BrainEx are improved and developed for use in humans, people who are declared brain dead (especially those with brain injuries resulting from a lack of oxygen) could become candidates for brain resuscitation rather than organ donation. Certainly, it could become harder for physicians or family members to be convinced that further medical intervention is futile“ [6, S. 302f]. Jede Diskussion um den Hirntod macht diese Situation nicht besser.

Aufwind hingegen werden die Firmen bekommen, die „Kryokonservierung“ von Verstorbenen oder nur von deren Köpfen verkaufen. Wenn man ein großes Gehirn heute 4 Stunden nach dem Tod (fast) wiedererwecken kann, warum sollte das dann in 200 Jahren nicht nach 200 Jahren im Gefrierfach gehen? Schon heute ist bei der Kühlungsbehandlung (Hypothermie) nach plötzlichem Herztod, anschließender Reparatur des Herzens und Wiedererwärmung vor allem das richtige Vorgehen und Timing beim Erwecken, nicht beim Herunterkühlen, von entscheidender Bedeutung [7]. Man wird also darüber mit Sicherheit weiterforschen. Was dabei herauskommt, wissen wir heute nicht. Alle sind sich einig, dass wir jetzt mehr darüber reden müssen. Das ist gut so. 
Der Autor gibt an, dass kein Interessenkonflikt besteht.

\section{Korrespondenzadresse}

Prof. Dr. Dr. Manfred Spitzer

Universität Ulm

Abteilung für Psychiatrie

Leimgrubenweg 12-14

89075 Ulm

\section{Literatur}

[1] Vrselja Z, Daniele SG, Silbereis ] et al. Restoration of brain circulation and cellular functions hours post-mortem. Nature 2019; 568: 336-343

[2] Putnam H. Vernunft, Wahrheit und Geschichte. Suhrkamp: Frankfurt; 1981/1982

[3] Farahany NA, Greely HAT, Giattino CM. Part-revived pig brains raise ethical quandires. Nature 2019; 568: 299-302

[4] Vrselja Z et al. Nature 2019; doi. org/10.1038/s41586-019-1099-1
[5] Reardon S. Pig brains kept alive for hours outside body. Nature 2019; 568: 283-284

[6] Youngner S, Hyun I. Pig brain Study could fuel debate around death. Nature 2019; 568: 302-304

[7] Evers M. Der Tod ist umkehrbar. Der Spiegel 2013; 30: 92-97

\section{Bibliografie}

DOI https://doi.org/10.1055/a-0873-8919 Nervenheilkunde 2019; 38: 418-421 